\title{
Explorando las relaciones entre el estrés y la situación económica en ciudadanos de Córdoba, Argentina*
}

\section{Exploring the relationships between stress and the economic situation in citizens from Córdoba, Argentina}

\author{
Débora Mola \\ ORCID: 0000-0002-7810-2424 \\ Pablo Correa \\ ORCID: 0000-0001-7597-7180 \\ Cecilia Reyna**
}

ORCID: 0000-0002-6097-4961

Instituto de Investigaciones Psicológicas,

Consejo Nacional de Investigaciones

Científicas y Técnicas (IIPSI-CONICET), Universidad Nacional de Córdoba, Argentina

Recibido: 28 de mayo de 2018 Revisado: 26 de julio de 2018 Aceptado: 14 de noviembre de 2018

\section{Resumen}

El estrés afecta nuestra percepción del mundo y también nuestro comportamiento, pero parece no afectar a todos por igual, sino que esta influencia varía según factores tales como el estatus económico. A partir de eso, en este estudio analizamos las diferencias en los niveles de estrés percibido según el sexo y el estatus socioeconómico, y su relación con la percepción de la situación económica tanto de la familia como del país, en una muestra de ciudadanos de Córdoba, Argentina. La muestra comprendió 279 personas de 18 a 65 años $(M=36.61, D S=13.97)$. Se utilizaron cuestionarios estructurados para evaluar las distintas variables de interés. Los resultados evidenciaron diferencias en los niveles de estrés percibido según el estatus económico, aunque la magnitud fue baja. A su vez, se observaron relaciones con la percepción de la situación económica, particularmente de la familia, con un tamaño del efecto de pequeño a moderado. Se subraya la necesidad de contar con nueva evidencia empírica sobre los determinantes y consecuencias del estrés.

Palabras clave: estrés percibido, estatus económico, percepción de la situación económica

Artículo de investigación. El presente trabajo ha sido financiado por la Agencia Nacional de Promoción Científica y Tecnológica [subsidio número PICT2014-1835]. Cómo citar: Mola, D., Correa, P., \& Reyna, C. (2019). Explorando las relaciones entre el estrés y la situación económica en ciudadanos de Córdoba, Argentina. Diversitas: Perspectivas en Psicología, 15(2), 251-256. DOI: 10.15332/22563067.4287

Correspondencia: Cecilia Reyna, Instituto de Investigaciones Psicológicas, Consejo Nacional de Investigaciones Científicas y Técnicas (IIPSICONICET), Universidad Nacional de Córdoba, Argentina. Dirección postal: Enrique Barros y Enfermera Gordillo, Ciudad Universitaria, 5000 Córdoba, Argentina. Correo electrónico: ceciliareyna@unc.edu.ar 


\section{Abstract}

Stress affects our perception of the world and our behavior, but it does not seem to affect everyone equally, instead that influence varies according to factors such as economic status. Taking this into account, in this study we analyze the differences in the levels of perceived stress according to sex and socioeconomic status, and its relationship with the perception of the economic situation of both the family and the country, in a sample of citizens from Córdoba Argentina. The sample comprised people from 18 to 65 years old $(M=36.61, D S=13.97)$. Structured questionnaires were used to evaluate the different variables of interest. The results showed differences in perceived stress levels according to economic status, although the magnitude was low. In turn, relationships were observed with the perception of the economic situation, particularly of the family, with a small to moderate effect size. The need to have new empirical evidence on the determinants and consequences of stress is underlined.

Keywords: perceived stress, economic status, perception of the economic situation

\section{Introducción}

Un reporte reciente de la American Psychological Association (2017) señala que niveles más altos de estrés entre poblaciones de bajos ingresos y minorías pueden dar lugar a disparidades en salud, lo cual afecta la expectativa de vida. El estrés afecta nuestra percepción del mundo y, en consecuencia, nuestro comportamiento.

El estrés es definido como el grado en que una situación puede ser valorada como incontrolable, impredecible y agobiante (Baik et al., 2019; Cohen, Kamark, \& Mermelstein, 1983). Se lo concibe también como una respuesta ante una situación temida (Barbosa-Leiker et al., 2012) que sobrepasa los recursos de afrontamiento de una persona (González Ramírez \& Landero Hernández, 2007), impactando negativamente sobre su salud (Cázares de León \& Moral de la Rubia, 2016).

El estrés percibido (particularmente, evaluado con la Escala de Estrés Percibido de Cohen et al., 1983) se ha relacionado con distintas variables sociodemográficas, como el sexo y el estatus socioeconómico. En cuanto al sexo, diferentes estudios evidenciaron que las mujeres reportan mayores niveles de estrés percibido que los hombres (Alimar, Benítez, Koch, \&
Noel, 2013; Barbosa-Leiker et al., 2012; Faro, 2015; Klein et al. 2016; Lee, Chung, Suh, \& Jung, 2014; Nordin \& Nordin, 2013; Remor, 2006; Sacco, Bucholz, \& Harrigton, 2014). No obstante, en otras investigaciones no se han observado diferencias significativas según el sexo (Cázares de León \& Moral de la Rubia, 2017; Cohen et al., 1983; González-Ramírez \& Landero-Hernández, 2007; Taylor, 2015).

Respecto al estatus socioeconómico, Faro (2015) observó que las personas de Brasil con ingreso económico alto (superior a 2000 reales, equivalente a 493 USD a la fecha) reportaron menor nivel de estrés percibido que las personas con ingresos bajos. A su vez, Baik et al. (2019) encontraron en una muestra de habitantes de Estados Unidos que la percepción del estrés depende de la interacción entre ingreso económico y sexo. Concretamente, las personas con ingresos bajos indicaron mayores niveles de estrés percibido, y los hombres con ingresos bajos reportaron mayores niveles de estrés que las mujeres con el mismo nivel de ingreso.

El estrés no sólo se ha relacionado con medidas objetivas cuantificables en relación con el de nivel socioeconómico sino también con la percepción de la situación económica. Por ejemplo, Berger, Powell 
y Cook (1988) evaluaron la relación entre el estrés auto-percibido y la percepción de bienestar económico en personas que se habían mudado y hallaron que las personas reportaban mayores niveles de estrés al percibir que su situación económica actual era peor que la que poseían antes de mudarse.

Teniendo en cuenta el rol del estrés en distintas esferas de la vida cotidiana, nos propusimos indagar las diferencias según el sexo y el estatus socioeconómico, y también su relación con la percepción de la situación económica tanto de la familia como del país, en una muestra de ciudadanos de Córdoba, Argentina.

\section{Método}

\section{Participantes}

Participaron 279 personas de 18 a 65 años de la ciudad de Córdoba $(M=36.61, D S=13.97)$ de ambos sexos ( $49.8 \%$ varones y $50.2 \%$ mujeres). Se utilizó un muestreo polietápico por conglomerados y estratificado (sexo y edad), preservando el principio de selección probabilística. La unidad primaria de muestreo fueron los hogares y la segunda las personas. El nivel de confianza fue del $95.5 \%$, con $\pm 5 \%$ de margen de error. El nivel socioeconómico (definido a partir de indicadores educativos, laborales y del hogar) de los participantes fue el siguiente: $17.8 \%$ marginal y bajo inferior, $23.6 \%$ bajo superior, $23.6 \%$ medio bajo, $21.5 \%$ medio, $13.5 \%$ medio alto y alto.

\section{Aspectos éticos}

A lo largo de toda la investigación se siguieron los lineamientos éticos para la investigación con humanos recomendados por la Asociación Psicológica Americana (APA, 2010) y los aspectos éticos considerados en la legislación local (FEPRA, 2013). El proyecto macro en el que se inserta esta investigación fue aprobado en cuanto a aspectos éticos. Los participantes brindaron su consentimiento informado de manera oral, tras recibir información sobre las condiciones de anonimato, confidencialidad y voluntariedad de la participación.

\section{Instrumentos}

\section{Escala de estrés percibido (Cohen et al., 1983).}

La escala comprende 14 ítems que permiten identificar en qué medida se evalúan como estresantes distintas situaciones de la vida. Los ítems de la escala se puntúan en una escala tipo Likert de 5 puntos ( $0=$ nunca, $4=$ muy frecuentemente). La puntuación total indica que a una mayor puntuación corresponde un mayor nivel de estrés percibido. Estudios psicométricos de la escala en el contexto local evidencian buenas propiedades de confiabilidad (Alfa de Cronbach = .788; Reyna, Mola, \& Correa, 2019).

\section{Percepción de la situación económica del país y la familia.}

Se utilizaron preguntas del Latinobarómetro (2015). Específicamente, la valoración de la situación económica del país y de la familia, tanto actual, como de los últimos y también próximos 12 meses. El formato de respuesta fue tipo Likert con 5 opciones de respuesta $(1=$ muy mal o mucho peor, $5=$ muy buena o mucho mejor).

\section{Cuestionario de datos sociodemográficos.}

Se recabó información sobre edad, sexo y variables indicadoras del nivel socioeconómico (NSE): nivel educativo, tipo de ocupación, relación entre la cantidad de aportantes del hogar y la cantidad de habitantes (Comisión de Enlace Institucional, AAMSAIMO- CEIM, 2006).

\section{Procedimiento}

Este estudio forma parte de una investigación macro sobre efectos de la pobreza y la desigualdad en variables cognitivas y sociales. En este reporte nos centramos en el estrés percibido. La recolección de datos estuvo a cargo de encuestadores previamente entrenados por una de las investigadoras, y se realizó entre noviembre y diciembre de 2015. 
Los encuestadores contactaron a las personas en sus hogares y les aplicaron individualmente los cuestionarios. Los cuestionarios fueron aplicados siempre en el mismo orden. Llevó 40 minutos completar todos los cuestionarios de la investigación marco.

\section{Análisis de datos}

Inicialmente se analizaron los datos de manera descriptiva y se evaluó si seguían una distribución normal. Luego se condujeron análisis de comparación de grupo (según NSE y sexo) y de correlación (entre estrés y percepción de la situación económica). Se consideró un nivel de significancia de .05 y se tuvo en cuenta el tamaño del efecto.

\section{Resultados}

\section{Análisis descriptivo}

Los valores promedio de estrés percibido estuvieron en un rango de 1.29 a 4.39 puntos, con una $M=2.77$ y $D S=.53$. La variable presentó valores de asimetría y curtosis en el rango \pm 1 . La prueba de KolmogorovSmirnov evidenció que la variable se distribuía de manera normal $(Z$ de $K-S=1.007, p=.263)$.

\section{Diferencias de estrés percibido según nivel socioeconómico y sexo}

Se analizaron las diferencias de sexo y NSE a través de ANOVAs, considerando como variable dependiente a la puntuación total de estrés percibido. Los resultados evidenciaron un efecto principal de NSE $\left(F(4,265)=5.550, p<.001, \eta_{p}^{2}=\right.$ .077). Comparaciones a posteriori con ajuste de Bonferroni muestran que las personas de nivel bajo y bajo inferior $(M=3.04, D S=.51)$ presentan niveles de estrés percibido significativamente mayores que las personas de nivel medio $(M=2.60, D S=.46 ; p$ <.001), y medio alto y alto $(M=2.65, D S=.41 ; p=$ .006). No se apreciaron diferencias según el sexo, y tampoco resultó significativa la interacción entre Sexo y NSE.

\section{Relación entre el estrés percibido y la percepción de la situación económica del país y de la familia}

Teniendo en cuenta las diferencias según NSE, para examinar las relaciones bivariadas entre el nivel de estrés percibido y la percepción de la situación económica del país/familia, se condujeron análisis de correlación parcial. El nivel de estrés no se relacionó de manera significativa con la percepción económica del país actual ni de los últimos meses, pero sí con la percepción de la situación futura $(r=$ $-.120, p=.048$ ), quienes mostraron una percepción de estrés mayor evaluaron con más negativa la situación económica del país para los próximos 12 meses. A su vez, la percepción del estrés se relacionó con la percepción de la situación económica de la propia familia. Específicamente, quienes presentaron niveles más altos de estrés percibido consideraron de manera más negativa la situación económica actual de su familia $(r=-.256, p<.001)$, y también en relación al último $(r=-.155, p=.011)$ y próximo año $(r=-.174, p=.004)$.

\section{Discusión}

En esta investigación analizamos la relación entre el nivel de estrés percibido y distintas variables sociodemográfica, como también con la percepción de la situación económica del país y de la propia familia.

Los resultados mostraron que el estrés percibido no variaba según el sexo de los participantes, aportando evidencia en la línea de Cohen et al. (1983) y Taylor (2015), entre otros. Con respecto al nivel socioeconómico, se observó que las personas de nivel socioeconómico alto reportan menores niveles de estrés percibido en comparación con las personas de niveles socioeconómicos bajos y medios. Estos resultados están en consonancia con lo observado en estudios previos con muestras de otras latitudes (p. e., Baik et al., 2019; Faro, 2015). No obstante, vale señalar que el tamaño del efecto fue pequeño. 
En cuanto a la relación entre el nivel de estrés percibido y la percepción de la situación económica, los resultados mostraron una relación significativa cuando la percepción de la situación económica se refería a la propia familia. En todos los casos, la magnitud de las relaciones fue de pequeña a mediana, siendo un poco más fuerte cuando se hacía referencia a la situación económica actual. Estos resultados son coincidentes con los reportados por Berger et al. (1988) quien también encontraron que las personas reportaban mayores niveles de estrés al percibir su situación económica actual como peor a la que poseían antes de mudarse.

A través de este estudio aportamos evidencia sobre las diferencias de estrés percibido según el nivel socioeconómico, y también su relación con la percepción de la situación socioeconómica, principalmente, familiar. Considerando el impacto negativo del estrés sobre diversos comportamientos (p.e., relacionados con la salud), resulta imperioso conducir nuevos estudios que aporten evidencia sobre las consecuencias del estrés y también los factores determinantes y, así, contar con evidencia científica que permita emprender estrategias para mitigar los niveles de estrés, en especial, en personas que viven en condiciones de vulnerabilidad socioeconómica.

\section{Referencias}

American Psychological Association. (2010). Ethical principles of psychologist and code of conduct. Washington, DC: APA

American Psychological Association, APA Working Group on Stress and Health Disparities. (2017). Stress and health disparities: Contexts, mechanisms, and interventions among racial/ethnic minority and low-socioeconomic status populations. Recuperado de http://www.apa.org/pi/ health-disparities/resources/stress-report.aspx

Baik, S. H., Fox, R. S., Mills, S. D., Roesch, S. C., Sadler Robins, G., ... Malcarne, V. L. (2019). Reliability and validity of the Perceived Stress Scale-10 in Hispanic Americans with English or Spanish language preference.
Journal of Health Psychology, 628-639. DOI: 10.1177/1359105316684938

Barbosa-Leiker, C., Kostick, M., Lei, M., McPherson, S., Roper, V., ... Wright, B. (2012). Measurement invariance of the Perceived Stress Scale and latent mean differences across gender and time. Stress and Health, 29, 253-260. DOI: 10.1002/smi.2463

Benítez, A., Koch, S., \& Noel, G. (2013). Validación de la escala de estrés percibido (EEP-13) en una muestra de funcionarios públicos venezolanos. Anales de la Universidad Metropolitana, 13(1), 117-133. https://dialnet.unirioja.es/descarga/ articulo/4391585.pdf

Berger, P. S., Powell, J., \& Cook, A. (1988). The relation of economic factors to perceived stress in mobile families. Lifestyles: Family and Economic Issues, 9(4), 297-313. DOI: 10.1007/BF00986748

Cázares de León, F., \& Moral de la Rubia, J. (2016). Diferencias sociodemográficas y de práctica profesional asociadas a estrés percibido entre dentistas colegiados de Monterrey. Avances en Psicología Latinoamericana, 34(1), 83-97. DOI: 10.12804/apl34.1.2016.06

Cohen, S., Kamarck, T., \& Mermelstein, R. (1983). A global measure of perceived stress. Journal of Health and Social Behavior, 24, 385-396. DOI: 10.2307/2136404

Comisión de Enlace Institucional, AAM-SAIMO-CEIM (2006). Nivel Socio Económico 2006. Recuperado de http://www.saimo.org.ar/socios/Socios/ NSE2006-23nov2006-Informe_final.pdf

Faro, A. (2015). Confirmatory factor analysis of three versions of the Perceived Stress Scale (PSS): A population-based study. Psicologia: Reflexão e Crítica, 28(1), 21-30. DOI: 10.1590/1678-7153.201528103

Federación de Psicólogos de la República Argentina (2013). Código de Ética. Recuperado de http:// fepra.org.ar/docs/C_ETICA.pdf 
González Ramírez, M. T. \& Landero Hernández, R. (2007). Factor structure of the Perceived Stress Scale (PSS) in a sample from Mexico. The Spanish Journal of Psychology, 10(1), 199-206. DOI: $10.1017 /$ S1138741600006466

Klein, E. M., Brähler, E., Dreier, M., Reinecke, L., Müller, K. W., ... Beutel, M. E. (2016). The German version of the Perceived Stress Scale Psychometric characteristics in a representative German community sample. BMC Psychiatry, 16, 159. DOI: 10.1186/s12888-016-0875-9

Latinobarómetro (2015). Cuestionario 2015. Recuperado de http://www.latinobarometro. org/latContents.jsp

Lee, E., Chung, B. Y., Suh, C., \& Jung, J. (2014). Korean versions of the Perceived Stress Scale (PSS-14, 10 and 4): Psychometric evaluation in patients with chronic disease. Scandinavian Journal of Caring Sciences, 29, 183-192. DOI: 10.1111/scs.12131

Nordin, M. \& Nordin S. (2013). Psychometric evaluation and normative data of the Swedish version of the 10 -item perceived stress scale. Scandinavian Journal of Psychology, 54, 502507. DOI: 10.1111/sjop.12071
Remor, E. (2006). Psychometric properties of a European Spanish version of the Perceived Stress Scale (PSS). The Spanish Journal of Psychology, 9(1), 86-93. DOI: 10.1017/ S1138741600006004

Reyna, C. E., Mola, D. J., \& Correa, P. S. (2019). Escala de Estrés Percibido: Análisis Psicométrico desde la TCT y la TRI. Revista de Ansiedad y Estrés. DOI: 10.1016/j.anyes.2019.04.003

Sacco, P., Bucholz, K. K., \& Harrington, D. (2014). Gender differences in stressful life events, social support, perceived stress, and alcohol use among older adults: Results from a National Survey. Substance Use and Misuse, 49(4), 456465. DOI: 10.3109/10826084.2013.846379

Taylor, J. (2015). Psychometric analysis of the ten-item Perceived Stress Scale. Psychological Assessment, 27(1), 90-101. DOI: 10.1037/a0038100 